\title{
Novel GANAB variants associated with polycystic liver disease
}

\author{
Liyanne F. M. van de Laarschot ${ }^{1}$, René H. M. te Morsche' ${ }^{1}$, Alexander Hoischen², Hanka Venselaar ${ }^{3}$, \\ Hennie M. Roelofs ${ }^{1}$, Wybrich R. Cnossen ${ }^{1}$, Jesus M. Banales ${ }^{4,5,6}$, Ronald Roepman ${ }^{2 \dagger}$ and Joost P. H. Drenth ${ }^{1 *+}$
}

\begin{abstract}
Background: Polycystic liver disease (PLD) is an inherited disorder characterized by numerous cysts in the liver. Autosomal dominant polycystic kidney and liver disease (ADPKD and ADPLD, respectively) have been linked to pathogenic GANAB variants. GANAB encodes the a-subunit of glucosidase II (Glla). Here, we report the identification of novel GANAB variants in an international cohort of patients with the primary phenotype of PLD using molecular inversion probe analysis.

Results: Five novel GANAB variants were identified in a cohort of 625 patients with ADPKD or ADPLD. In silico analysis revealed that these variants are likely to affect functionally important domains of glucosidase II a-subunit. Missense variant C.1835G >C p.(Arg612Pro) was predicted to disrupt the structure of the active site of the protein, likely reducing its activity. Frameshift variant c.687delT p.(Asp229Glufs*60) introduces a premature termination codon predicted to have no activity. Two nonsense variants (c.2509C > T; p.(Arg837*), and c.2656C $>$ T; p. (Arg886*)) and splice variant c. $2002+1 G>C$, which causes aberrant pre-mRNA splicing and affecting RNA processing, result in truncated proteins and are predicted to cause abnormal binding of $\mathrm{a}$ - and $\beta$-subunits of glucosidase II, thus affecting its enzymatic activity. Analysis of glucosidase II subunits in cell lines shows expression of a truncated Glla protein in cells with c.687delT, c.2509C > T, c.2656C > T, and c.2002+1G >C variants. Incomplete colocalization of the subunits was present in cells with c.687delT or c.2002+1G>C variants. Other variants showed normal distribution of Glla protein.
\end{abstract}

Conclusions: We identified five novel GANAB variants associated with PLD in both ADPKD and ADPLD patients supporting a common pathway in cystogenesis. These variants may lead to decreased or complete loss of enzymatic activity of glucosidase II which makes GANAB a candidate gene to be screened in patients with an unknown genetic background.

Keywords: Polycystic liver disease, Glucosidase II, Molecular inversion probe analysis, Liver cysts, Cholangiocytes

\section{Background}

Development of numerous liver cysts is the primary phenotype of autosomal dominant polycystic liver disease (ADPLD). ADPLD patients may present with few

\footnotetext{
*Correspondence: joost.drenth@radboudumc.nl

${ }^{\dagger}$ Ronald Roepman and Joost P. H. Drenth should be considered joint senior author

1 Department of Gastroenterology and Hepatology, Institute for Molecular Life Sciences, Radboud University Medical Center, P.O. Box 9101, 6500 HB Nijmegen, The Netherlands

Full list of author information is available at the end of the article
}

kidney cysts, but renal function remains preserved [1, 2]. On the contrary, kidney cysts are the dominant feature of patients with autosomal dominant polycystic kidney disease (ADPKD), and the majority of these patients develop concurrent liver cysts [3]. Almost all ADPKD patients harbour gene mutations in polycystic kidney disease 1 (PKD1) or polycystic kidney disease 2 (PKD2) [4]. The genetic landscape in ADPLD is more diverse as, to date, mutations in at least seven genes explain collectively only $25-30 \%$ of the genetic spectrum of ADPLD $[2,5]$. Although ADPLD and ADPKD are two distinct genetic 
disorders, they share PLD as a major phenotypic feature. Recent evidence suggests that the involved proteins interact, with decreased levels of functional polycystin-1 (PC1), encoded by PKD1, as the central element for cyst development $[6,7]$.

With the exception of LRP5 (a transmembrane protein part of the LRP5/LRP6/Frizzled co-receptor complex [8, 9]) all genes involved in ADPLD encode proteins involved in endoplasmic reticulum trafficking and quality control of glycoproteins. These genes include PRKCSH, SEC63, GANAB, ALG8, SEC61B, DNAJB11, and ALG9 [10]. Central to the pathomechanism of ADPLD is glucosidase II, an endoplasmic reticulum (ER) resident N-linked glycanprocessing enzyme. Glucosidase II (GII) is a complex of catalytic $\alpha$-subunit (GII $\alpha$ ), encoded by GANAB, and a regulatory $\beta$-subunit (GII $\beta$ ), encoded by PRKCSH. Collectively this contributes to the localization and enzymatic activity of GII [11]. Glucosidase II catalyzes the two-step hydrolysis at $\alpha 1,3$-linked glucose-glucose and glucose-mannose residues of high-mannose-type glycans to generate a quality control protein tag on glycoproteins that is recognized by ER chaperones [12]. As such, it acts as a major partner in glycoprotein processing and quality control in the ER [13].

The concept that genetic defects disrupting different subunits of a protein complex result in a very similar phenotype ("guilt by association") is well-accepted [14]. While mutations in GII $\beta$ have been associated with ADPLD, the phenotypic picture for GII $\alpha$ is less clear. Most studies have linked GANAB to ADPKD and renal cysts were present in most patients [15]. We set out to explore the incidence of GANAB mutations in a large, independent cohort of patients with PLD as dominant phenotype, and to evaluate the effect of GANAB mutations on protein structure and glucosidase II subunit binding.

\section{Results \\ Patient characteristics}

For our molecular inversion probe (MIP) analysis we included a total of 625 patients with ADPKD or ADPLD. Patients were referred to our center for genetic analysis or treatment of PLD by hospitals from the Netherlands, Belgium, Spain, and Denmark. All patients had the primary phenotype of polycystic liver disease (PLD). Patients were diagnosed with ADPKD or ADPLD based on clinical presentation. 90 patients were diagnosed with ADPKD, and 535 patients with ADPLD. Of the 625 analysed patients, 17 families were included that consisted of 62 family members. Patients had not been genetically screened previously.

Of the eight patients with likely pathogenic heterozygous GANAB variants, seven patients presented with ADPLD and three patients had 1-3 kidney cysts, while in the remaining patients no kidney cysts could be detected. One patient was diagnosed with ADPKD. The hepatic phenotype did not differ between patients affected by ADPLD or ADPKD. Six out of eight patients were female and the mean age was 56 (range 31-79) years. Clinical presentation of patients with GANAB variants can be found in Table 1.

Table 1 Clinical presentation of kidney and liver disease in 8 affected individuals with GANAB variants

\begin{tabular}{|c|c|c|c|c|c|c|c|c|}
\hline & \multicolumn{2}{|l|}{ Family 11-0741 } & \multicolumn{6}{|c|}{ Unrelated individuals } \\
\hline & 8951 & 9087 & 8380 & 11726 & 11475 & 11716 & 8700 & 12241 \\
\hline Gender & Female & Female & Male & Female & Male & Female & Female & Female \\
\hline Diagnosis & ADPLD & ADPLD & ADPLD & ADPLD & ADPLD & ADPKD & ADPLD & ADPLD \\
\hline \multicolumn{9}{|c|}{ Mutation (RefSeq NM_198335) } \\
\hline $\begin{array}{l}\text { Chromo- } \\
\text { some } \\
\text { position } \\
\text { (Hg19) }\end{array}$ & g.62400926TG>G & g.62400926TG $>$ G & g.62393841C>T & g.62394111C>T & g.62396665G>C & $\begin{array}{l}\text { g.62414056delTAG } \\
\text { CGG }\end{array}$ & g.62397125G>C & g.62397125G>C \\
\hline $\begin{array}{l}\text { CDNA } \\
\text { change }\end{array}$ & c.687delT & c.687delT & c. $2656 C>T$ & c. $2509 C>T$ & c. $2002+1 G>C$ & c.11_16delTAGCGG & c. $1835 G>C$ & C. $1835 \mathrm{G}>\mathrm{C}$ \\
\hline $\begin{array}{l}\text { Amino acid } \\
\text { change }\end{array}$ & $\begin{array}{l}\text { p.Asp- } \\
\text { 229Glufs*60 }\end{array}$ & $\begin{array}{l}\text { p.Asp- } \\
\text { 229Glufs*60 }\end{array}$ & p.Arg886* & p.Arg837* & Splicing variant & p.Val4_Ala5del & p.Arg612Pro & p.Arg612Pro \\
\hline \multicolumn{9}{|c|}{ Radiological presentation } \\
\hline Type & $C T$ & Ultrasound & $C T$ & $C T$ & $\mathrm{n} / \mathrm{a}$ & MRI & $C T$ & $C T$ \\
\hline Liver cysts & $>20$ cysts & multiple cysts & 15 cysts & $>20$ cysts & $\mathrm{n} / \mathrm{a}$ & $>20$ cysts & $>10$ cysts & $>30$ cysts \\
\hline $\begin{array}{r}\text { kidney } \\
\text { cysts }\end{array}$ & No & No & 2 bilateral cysts & No & $\mathrm{n} / \mathrm{a}$ & $>20$ bilateral cysts & $\begin{array}{l}3 \text { cysts in left } \\
\text { kidney }\end{array}$ & 1 cyst in left kidney \\
\hline
\end{tabular}

$A D P K D$ autosomal dominant polycystic kidney disease, $A D P L D$ autosomal dominant polycystic liver disease, $C T$ computed tomography, $M R I$ magnetic resonance imaging, $n / a$ not available for review 


\section{Identification of GANAB variants in PLD-affected individuals and families}

MIP analysis identified 38 variants in 32 patients, of which 13 heterozygous GANAB variants are possibly pathogenic. We identified one frameshift, one in-frame deletion, one splice site, two nonsense, and eight missense variants that were all validated by Sanger sequencing (Fig. 1, Additional File 1). Notably, none of these variants have been reported before to cause ADPLD or ADPKD [15]. Moreover, registry data show that in-frame deletion c.11_16delTAGCGG (rs750723025) has a global frequency of 9/249,084 exomes $(\mathrm{MAF}=0.000036$; GnomAD) and is seen in $1 / 133,716$ European exomes $(\mathrm{MAF}=0.000007)$. The c. $2656 \mathrm{C}>\mathrm{T}$ variant (rs1210158408) is detected in $1 / 251,486$ exomes (MAF $=0.000003976$; GnomAD). The c.323C $>\mathrm{T}$ variant ( $\mathrm{rs} 200232092)$ is detected in $7 / 2,541,458$ exomes $(\mathrm{MAF}=0.000028$; GnomAD). The other variants are previously unknown and no allele frequency is available.

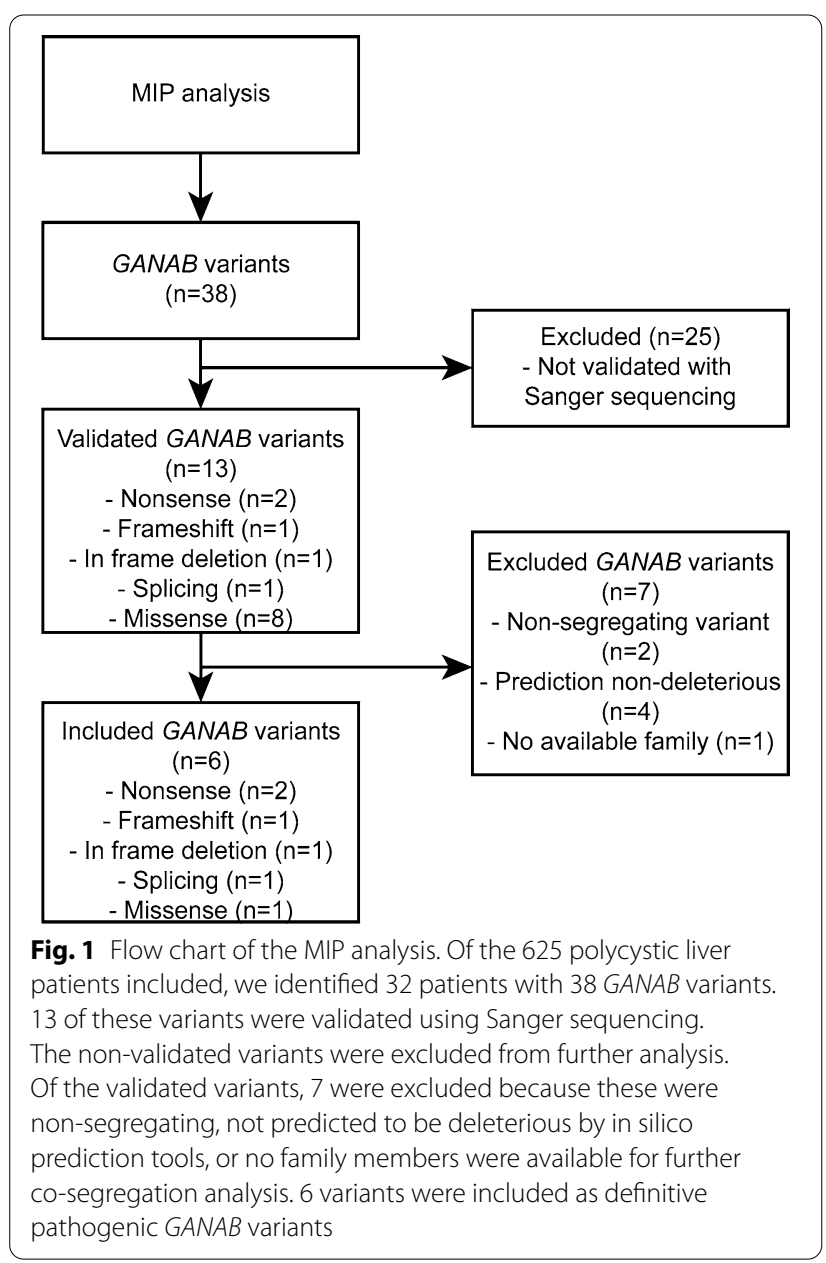

\section{Segregation analysis of GANAB variants}

To bolster the causal relationship between $G A N A B$ variants identified by MIP analysis and clinical presentation of PLD, we tested DNA of family members for GANAB variants. We confirmed that frameshift variant c.687delT co-segregated with the disease in family 11-0741. This ADPLD family presented with multiple liver cysts, without renal cysts which suggests that the variant is likely pathogenic.

For the patients with splice variant (c.2002+1G>C), and nonsense variants (c.2509C $>\mathrm{T}$ and c.2656C $>\mathrm{T}$ ) no DNA of family members was available for segregation analysis. Due to the predicted severe nature of these variants we included them in our final group of likely pathogenic variants.

Of the 8 missense variants, four variants (c. $1852 \mathrm{C}>\mathrm{T}$, c. $2006 \mathrm{~A}>\mathrm{G}$, c.38G >A, c.1835G >C) were predicted as deleterious by four distinct in silico predictors.

We analyzed two missense variants (c.1852C>T and c. $2006 \mathrm{~A}>\mathrm{G}$ ) predicted as deleterious, for segregation in affected family members. Missense variant c.1852C $>\mathrm{T}$ was present in the affected individual and her unaffected daughter. We did not identify a pathogenic $G A N A B$ variant in DNA derived from the affected brother of affected individual nor were mutations in other known genes for ADPLD present. Similarly, for the individual having the c. $2006 \mathrm{~A}>\mathrm{G}$ mutation both affected and unaffected family members possessed the $G A N A B$ variant. This suggests that neither variant c. $1852 \mathrm{C}>\mathrm{T}$ nor c. $2006 \mathrm{~A}>\mathrm{G}$ are causative for PLD in these families.

For the remaining two missense variants (c.38G $>\mathrm{A}$, c. $1835 \mathrm{G}>\mathrm{C}$ ) predicted as deleterious no family members were available for further analysis. However variant c.1835G >C p.(Arg612Pro) was reported in two unrelated individuals and is therefore included in the group of likely pathogenic variants.

Three missense variants (c.323C $>\mathrm{T}, \quad$ c.1607A $>\mathrm{C}$, c. $1883 \mathrm{C}>\mathrm{G}$ ) are predicted to be probably deleterious, the other variant $(\mathrm{c} .2702 \mathrm{C}>\mathrm{T})$ is predicted to be not deleterious by in silico prediction tools. These four variants were discarded as bona fide mutations due to the lack of available family members and the results of the prediction tools.

\section{MIP analysis of PRKCSH, SEC63 and PKD2}

We analyzed all patients with a validated likely pathogenic $G A N A B$ variant for a pathogenic sequence variation in PRKCSH, SEC63 and PKD2. None of the individuals who carried a bona fide pathogenic GANAB variant was trans heterozygous for any of the other three PLD associated genes. In the family with nonsegregating $G A N A B$ variant c. $2006 \mathrm{~A}>\mathrm{G}$, we identified 
the PRKCSH variant c. $841 \mathrm{C}>\mathrm{T}$ p.(Arg281Trp), which has previously been described to cause APDLD [16].

\section{Pathogenic GANAB variants influence glucosidase II structure in silico}

In order to predict the functional consequences of the identified $G A N A B$ variants, we evaluated their effects on the glucosidase II protein complex structure in silico. The relationship between the GANAB variants and the GII function and interaction of GII subunits was studied using 3D homology modelling of the GII protein complex. Structural changes caused by the GANAB variants were predicted and visualized. The complete protein structure and interaction sites of the two subunits of human GII have only recently been unraveled $[12,17]$. The GII protein complex consists of the $110 \mathrm{kDA}$ catalytic $\alpha$-subunit and the $60 \mathrm{kDA}$ regulatory $\beta$-subunit. GII $\alpha$ is composed of four major domains and three subdomains. The active site of GII $\alpha$ can be found in the $\beta_{8} \alpha_{8}$ barrel domain [12]. The distal C-terminal domain of GII $\alpha$ is primarily involved in binding of GII $\beta$ through its $\mathrm{N}$-terminal GII $\alpha$-binding domain [17]. The frameshift variant (c.687delT) is located at the N-terminal domain. Modelling predicts that this variant introduces a premature termination codon (PTC) 60 amino acids upstream (p.Asp229Glufs"60) leading to a truncated protein and thus limiting functional protein expression. In frame deletion p.Val4_Ala5del (c.11_16delTAGCGG) located to the signalling sequence, is predicted to result in degradation of the protein as the protein would be unable to be transported and mature. The amino acid substitution p.Arg612Pro in the $\beta_{8} \alpha_{8}$ barrel domain is the consequence of missense variant c. $1835 \mathrm{G}>\mathrm{C}$. This affects the structure of the domain carrying the active site of GII $\alpha$ and thus is predicted to reduce its activity. The two nonsense variants p.Arg837* (c.2509C $>\mathrm{T}$ ) and p.Arg886* (c.2656C $>\mathrm{T})$ result in a truncation of the distal C-terminal domain (Fig. 2). The last variant is located in a splice site region at the distal part of the gene, which is predicted to result in inaccurate premRNA splicing. Most likely this leads to a PTC resulting in a truncated protein.

\section{Glucosidase II a-subunit expression and localization in HEK293 and HeLa cells}

We transiently transfected the GANAB Wild Type (GANAB-WT) and the suspected pathogenic variants c.11_16delTAGCGG (GANAB-11), c.687delT (GANAB-687), c.2002+1G>C (GANAB-INT), c.2509C $>\mathrm{T}$ (GANAB-2509) and c.2656C $>\mathrm{T}$ (GANAB2656) constructs in HEK 293 and HeLa cells. Western blots using equal amounts of total protein from transiently transfected cells revealed expression of GII $\alpha$, truncated and non-truncated in HEK293 cells (Fig. 3a). GANAB-11 generated GII $\alpha$ with a normal appearing length. GANAB-687 resulted in truncated GII $\alpha$ of about $32 \mathrm{kDa}$ which is in line with the prediction of a 287 amino acid long truncated protein. GANAB-INT produced a truncated protein of about $80 \mathrm{kDa}$ suggesting the use of a stop codon starting $51 \mathrm{bp}$ upstream of exon 17. There were no quantitative expression differences which suggests there may be no nonsense-mediated RNA decay provoked by the presence of a PTC in variants GANAB-687 and GANAB-INT. GANAB2509 resulted in truncated GII $\alpha$ of about $92 \mathrm{kDa}$ and GANAB-2656 in a truncated protein of about $97 \mathrm{kDa}$. This is in line with the size of the predicted truncated protein. Experiments using HeLa cells produced similar results (Additional File 2). On the other hand, protein analysis of cells stably expressing the wild type and mutated intronic variant showed a $50 \%$ reduction of the truncated protein in cells (Fig. 3b) with an equal copy number of the incorporated gene (Additional File 3).

Immunofluorescent images of HeLa cells transfected with GANAB-WT showed normal distribution of GII $\alpha$ and co-localization with GII $\beta$ (Fig. 4a-c). Also, cells transfected with GANAB-11 showed a normal distribution of GII $\alpha$ and co-localization with GII $\beta$ which was in contrast to the predicted in silico analysis (Fig. 4d-f). Cells transfected with GANAB-687 (Fig. 4g-i) and GANAB-INT (Fig. 4j-l) demonstrated abberant GII $\alpha$ localization distributed throughout the cell or confined to the nucleus respectively. Co-localization with GII $\beta$ was hardly present probably due to absence of the C-terminal part of GII $\alpha$. Cells transfected with GANAB-2509 or GANAB-2656 showed

(See figure on next page.)

Fig. 2 Identification of GANAB variants and in silico prediction of effect of variants on Glla structure. a GANAB is located on chromosome $13 q 12.3$ and consists of 25 exons. The identified variants are located on exons 7, 16, 22, and 23. b Sequence electropherograms show heterozygous germline mutations c Predicted structure with p.Asp229Glufs*60 (c.687delT) variant resulting in early termination of the protein by introducing a premature stop codon. $\mathbf{d}$ p.Arg612Pro (c.1835G >C) variant results in amino acid change close to the active site of the protein. Affected amino acid is depicted in color. The inlay shows a magnification of the structural change of the amino acid. e Predicted structure with c.2509C $>$ T ( $p$.Arg837*) variant results in untranscribed distal C-terminal domain $\mathbf{f}$ Predicted structure with c.2656C $>T$ (p.Arg886*) variant results in untranscribed distal C-terminal domain. $\mathbf{c}, \mathbf{e}, \mathbf{f}$ Preserved protein is depicted in color, loss of protein structure in grey 
a

Chromosome 11

(q12.3)

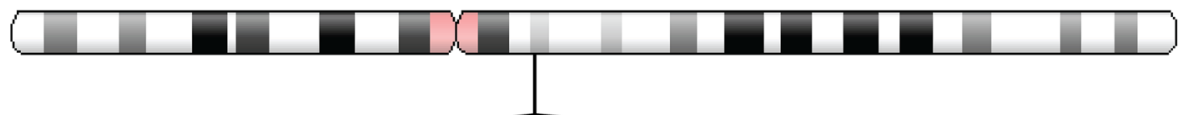

GANAB

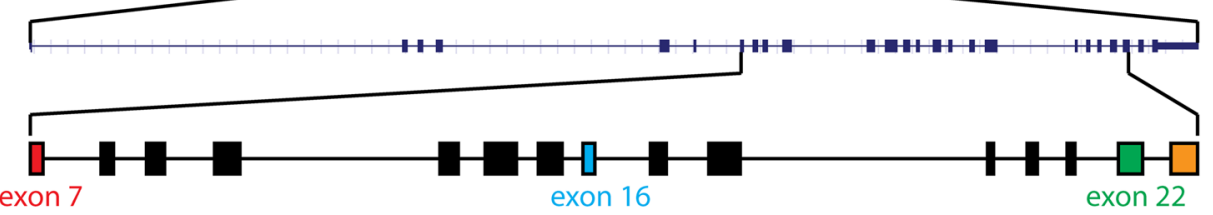

b

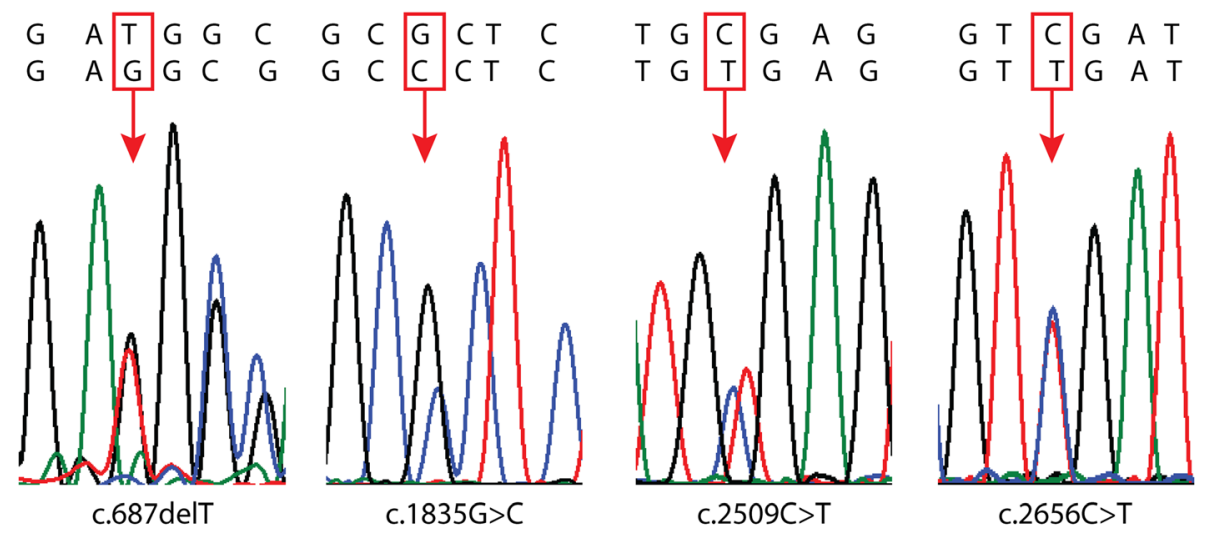

c

p.Asp229Glufs*60

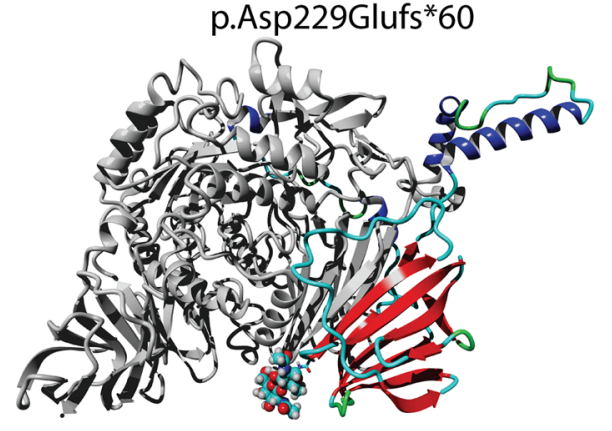

d

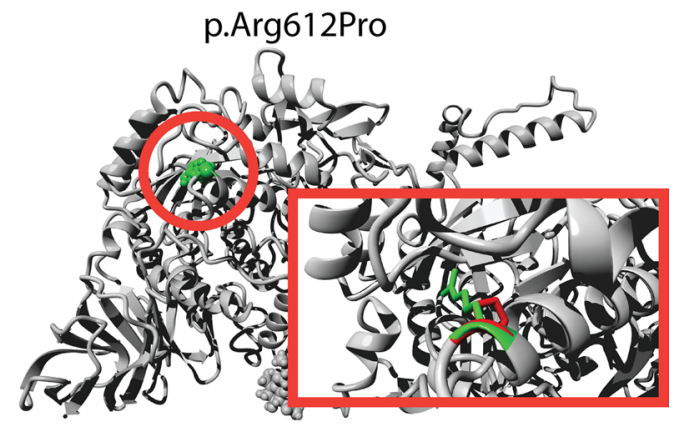

e

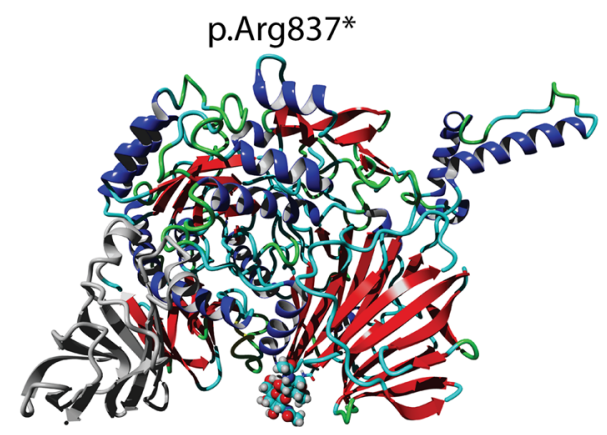

f

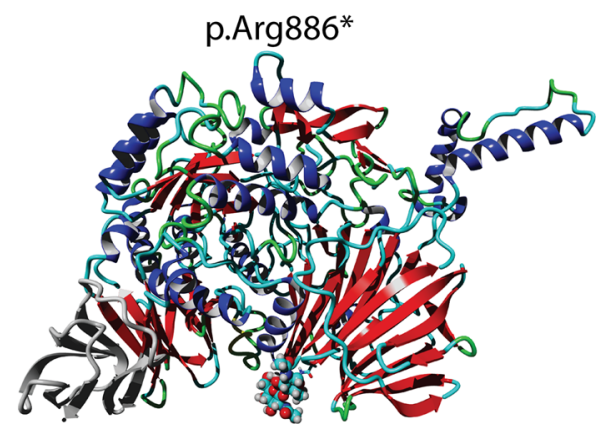




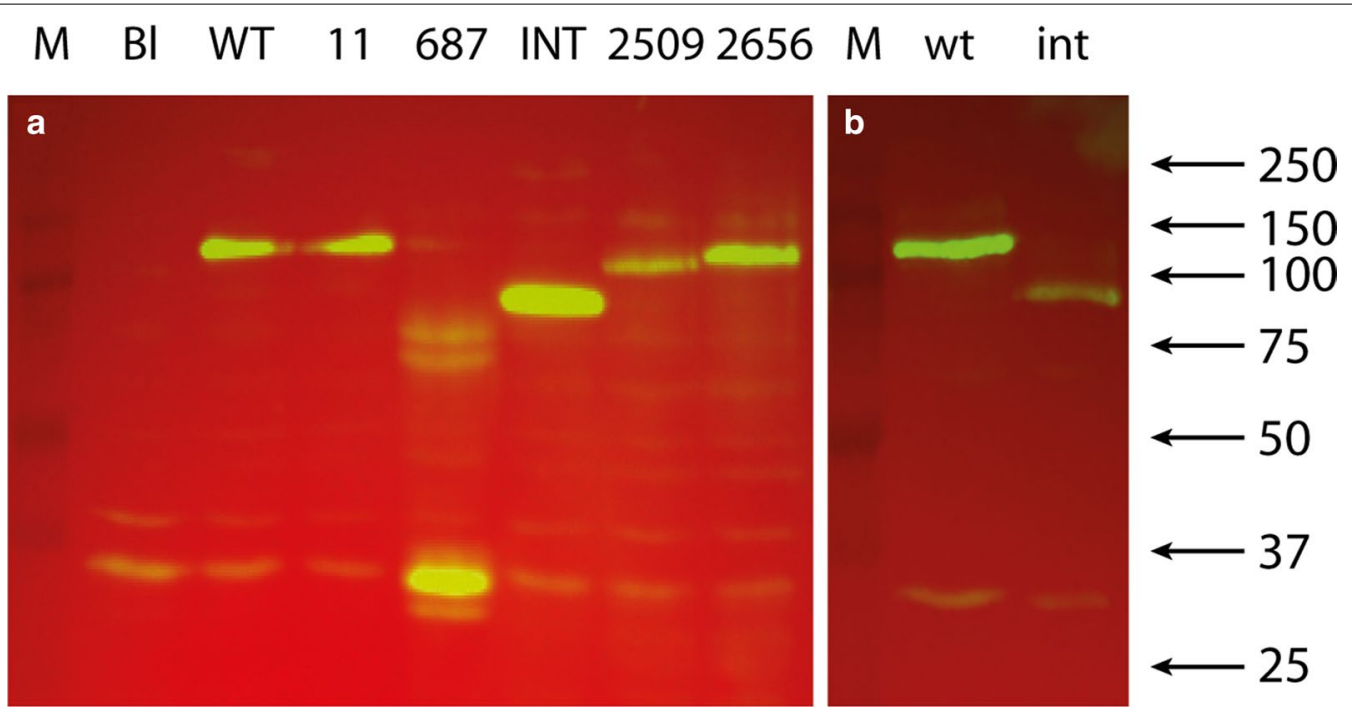

Fig. 3 Protein analysis of wild type and mutant Glla in HEK293 cells. Western blot detection using anti-Flag antibody of cell lysate from transiently transfected HEK293 cells using N-terminal Flag tagged constructs with: a GANAB Wild Type (WT) and variants c.11_16delTAGCGG (11), c.687delT (687) and c.2002+1G>C (INT) at te left of the marker (M). The constructs showed similar amounts of expressed Glla with predicted molecular weights; b stable transfected HEK293 cells resulted in reduced level of truncated Glla (INT) compared to the normal Glla (WT) using the intron construct

normal distribution of GII $\alpha$ despite the predicted partial absence of the C-terminal part of GII $\alpha$ (Additional File 4).

\section{Discussion}

Here we describe 5 novel GANAB variants that can be linked to the presence of PLD [15]. All patients presented severe PLD. Three ADPLD patients possessed 1-3 nonpathogenic kidney cysts, which is in concordance with the prevalence of kidney cysts in the general population [18]. These variants can cause PLD in patients diagnosed with ADPLD or ADPKD. Seven out of eight patients with a confirmed pathogenic GANAB variation in our cohort were diagnosed with ADPLD, the last patient had ADPKD.

Our in silico analysis suggests that the amino acid substitution in the $\beta_{8} \alpha_{8}$ barrel domain p.(Arg612Pro) probably results in reduced GII $\alpha$ enzymatic activity because the active site domain of the subunit is predicted to be disrupted. The variants c. $2656 \mathrm{C}>\mathrm{T}$ p. $($ Arg886*) and c. $2509 \mathrm{C}>\mathrm{T}$ p. $($ Arg837*) introduce a PTC that would lead to truncation of the C-terminal domain of GII . This could impair interaction with GII $\beta$ and consequently decrease enzymatic activity of GII $\alpha[17,19]$. This is in line with an interaction study showing that the Arg834Ala/ Arg835Ala mutation impairs interaction between GII $\alpha$ and GII $\beta$ subunits [17]. Further, from genetic studies the missense mutation in the distal C-terminal p.Arg839trp was identified to cause ADPKD in an ADPKD family
[15]. ER-localization of GII $\alpha$ is mediated by GII $\beta$ [20]. Our immunofluorescence assay did not show subcellular localization of GII $\alpha$. This would imply that variants c. $2656 \mathrm{C}>\mathrm{T}$ and c. $2509 \mathrm{C}>\mathrm{T}$ do not impair GII $\alpha$ and GII $\beta$ co-localization but may still affect enzymatic activity of GII $\alpha$.

The frameshift variant c.687delT p.(Asp229Glufs*60) and the splice variant c.2002+1G>C are likely to result in truncated proteins lacking the $\mathrm{C}$-terminal domain of GII $\alpha$ and can result in nonsense-mediated mRNA decay that reduces the level of translated protein expressed in the ER. However, no reduced levels of truncated proteins were seen in the transiently transfected cells compared to the wild type suggesting no influence of the PTCs on nonsense-mediated mRNA decay. Because transient transfection of cells can sometimes influence splicing and normal cellular processes due to overexpression of proteins we also performed stable transfection of the variants in which a reduced level of truncated protein of GANAB-INT was observed and therefore possibly indicates some, but not complete nonsense-mediated mRNA decay. Immunofluorescence assay shows no co-localization of both variants with GII $\beta$ and thus incomplete interaction which leads to reduced enzymatic of the GII complex.

Our study knows some limitations. Our study yielded a relative high number of non-validated GANAB variants. This was because all variants with a quality of depth of 500 or higher were included. This lower quality setting 


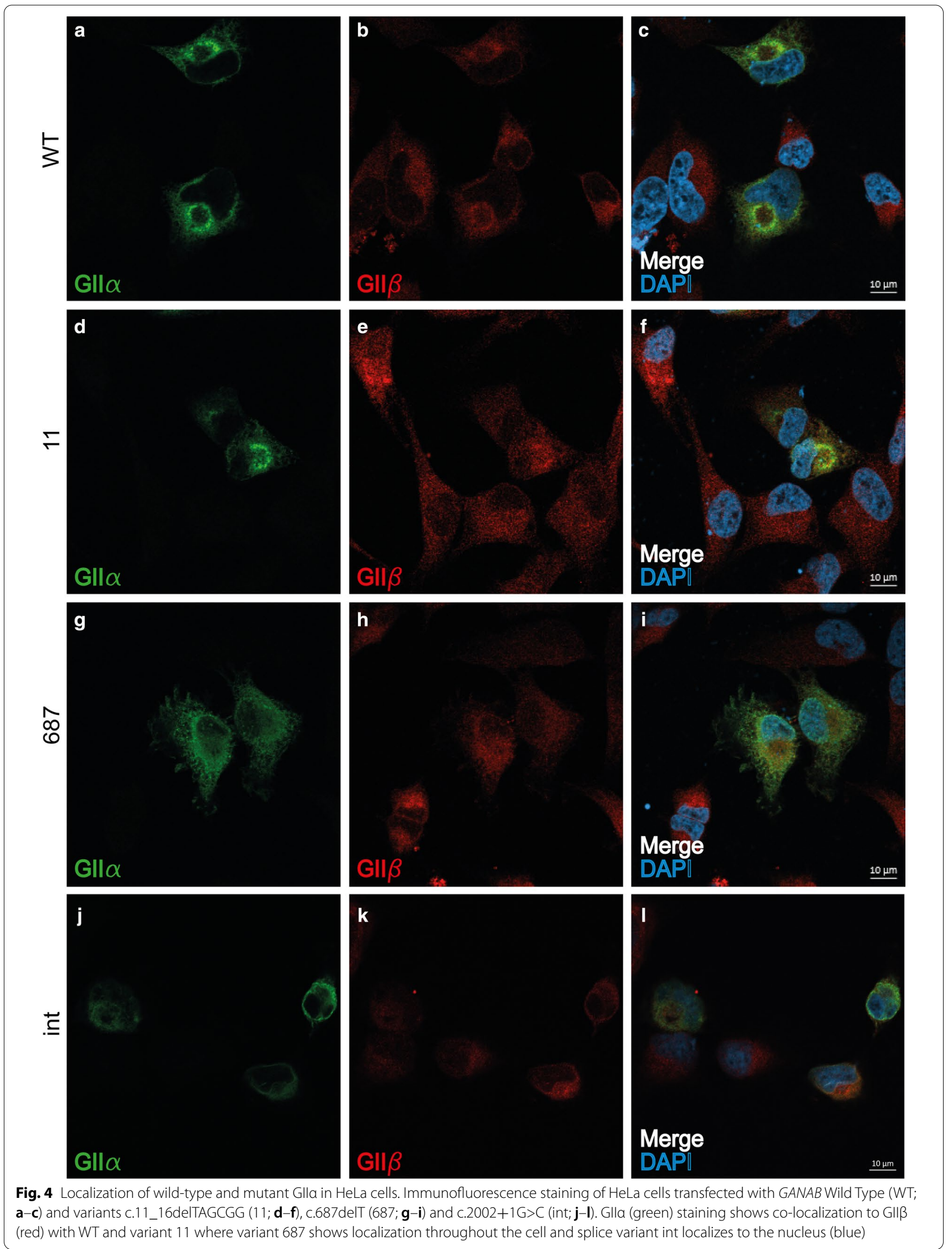


was chosen in order to not oversee any bona fide pathogenic variants in GANAB or the other three PLD genes. All cell studies were performed in HEK293 and HeLa cell lines. These cell lines are not specifically used as PLD models. Since the aim of our study was not to model PLD, but to study protein expression and localization of GII protein complex, these cell lines were chosen for their expression of both PRKCSH and GANAB. Cholangiocyte cell lines such as $\mathrm{H} 69$ or SkCHA-1 may be closer related to affected cells in PLD patients, but have low transfection rate and were thus deemed less suitable for our studies.

Overall, most identified variants will likely lead to decreased or complete loss of functional glucosidase II. Trimming of N-glycan glucose residues by glucosidase II is necessary for recognition by ER-resident chaperone proteins calnexin and calreticulin, and subsequent protein folding and secretion [21]. Therefore, functionality of glucosidase II is essential for correct protein maturation. We can hypothesize that defective glucosidase II directly affects polycystin-1 folding and maturation, and in this way causes cyst formation in the liver and/or kidney. However, as glucosidase II-driven protein maturation is not limited to polycystin-1, the absence of disease symptoms due to other disrupted cellular functions or organs remains unexplained. Also, it remains unclear what factors contribute to the differential expression of cysts in the liver and/or kidney. This should be investigated in future studies.

\section{Conclusions}

Our findings have two important implications. First, $G A N A B$ is the only gene that has been shown to cause polycystic liver disease in patients with ADPLD or ADPKD. This finding supports the standing hypothesis of a common pathway of hepatic cystogenesis in patients with ADPLD and ADPKD [22]. In vitro studies showed that mutations in genes associated with ADPLD result in defective maturation and trafficking of polycystin-1 $[7,15]$. The level of functional polycystin-1 at the cilium is postulated to be central in the development of both hepatic and kidney cysts [10]. If the level of functional polycystin-1 drops below a critical threshold, cyst development initiates. Furthermore, a more severe reduction of polycystin-1 function causes more severe cystic disease [6, 23]. Maturation of polycystin-1 is dependent on polycystin-2 chaperone function [24]. Moreover, the abundance of polycystin- 2 is determined by $\mathrm{N}$-glycosylation mediated by glucosidase II [25].

Second, our findings have some implications for clinical practice. Our cohort contains a large number of unrelated patients from different countries in Europe, which indicates that our results are a good reflection of the prevalence of GANAB variants in the European PLD patient population. In our study population the detection rate of bona fide pathogenic GANAB variants is $\sim 1 \%$. The detection rate in our total group of PLD-affected patients is low, nonetheless this number is similar to the detection rate of ADPLD associated gene LRP5 [9]. We suggest that genetic screening should include a screen for GANAB variants in ADPLD and ADPKD patients with unknown genetic background.

To conclude, we identified five novel GANAB variants associated with PLD in ADPKD and ADPLD patients. These variants may affect functionally important domains of GII and lead to decreased or complete loss of enzymatic activity of glucosidase II.

\section{Methods}

\section{Sample and data collection}

We collected clinical data and biomaterials from PLD patients in our PLD registry [26]. All participants of the PLD cohort provided informed consent for DNA analysis related to PLD studies. Blood samples were collected from patients and all available family members. Genomic DNA was isolated from the blood samples by standard methods with High Pure PCR Template Kit (Roche, Basel, Switzerland). Clinical and imaging data were obtained through review of clinical records.

\section{Editorial policies and ethical considerations}

This study was performed in line with the principles of the Declaration of Helsinki. Approval was granted by the Ethics Committee of the Radboud University Medical Center (CMO regio Arnhem-Nijmegen; 2001-218) and all participants gave informed consent.

\section{Molecular inversion probe analysis}

We used molecular inversion probe (MIP) analysis for DNA analysis of GANAB (RefSeq NM_198335), PRKCSH (RefSeq NM_001289104), SEC63 (RefSeq NM_007214), and PKD2 (RefSeq NM_000297). PRKCSH, SEC63, and $P K D 2$ were included in this analysis to exclude that PLD was caused by any of the known genes in these individuals. Due to technical difficulties caused by genomic duplicates in the first 34 exons, PKD1 was not included in the final MIP library. The final pooled MIP library comprised 174 probes $(G A N A B \mathrm{n}=54, P R K C S H \mathrm{n}=33$, SEC63 $\mathrm{n}=45, P K D 2 \mathrm{n}=42)$. The average coverage per probe was GANAB 1277, PRKCSH 263, SEC63 1369, PKD2 759. Capture regions were sequenced in two runs using 112-bp reads on a NextSeq500 sequencer (Illumina). MIP data variants were identified as described before [27]. Briefly, reads were aligned by Burrows-Wheeler Aligner to the reference genome. After MIP extension and ligation arms were removed from all the alignment 
files, reads were trimmed to remove overlap between the paired-ends. The identified variants were filtered on gene component (exon, acceptor site canonical, splice donor site canonical), synonymous (false), and zygosity (heterozygote) to exclude all false positive and non-changing variants. Variants that passed all filtering steps were validated using Sanger sequencing.

\section{Sanger sequencing}

Primers (Sigma-Aldrich, St Louis, MO) to amplify GANAB (25 exons), PRKCSH (18 exons), SEC63 (21 exons) and $P K D 2$ (15 exons) were designed using online software Primer3 (https://primer3.ut.ee/), and were used for PCR amplification (TL100 Biorad, Hercules, CA) of DNA samples. DNA bands were cut out of $1.5 \%$ agarose gel and purified using the QIAEXII kit (Qiagen, Hilden, Germany). Next, $10 \mathrm{ng}$ DNA per sample was used for Sanger sequencing. Sanger sequencing was performed at the Radboudumc Sequence Facility according to standard Sanger methods (3730 DNA Analyzer (Applied Biosystems, Foster City, CA)) with support of Big Dye Terminator ( v1.1 Thermofisher Scientific, Waltham, MA)).

Variants found with MIP analysis were compared to the obtained Sanger sequences with Alamut Visual (v2.7). The functional significance of novel mutations was assessed with bioinformatics prediction tools (PolyPhen-2, Align GVGD, SIFT, MutationTaster). Sanger sequencing was performed in all available family members for those patients with validated mutations. Validated variants were submitted to ClinVar database.

\section{In Silico analysis and homology modeling}

Homology models of the human GII $\alpha$ were created using the experimentally solved 3D-structure of the murine GII $\alpha$ as a template (Protein Data Bank file 5F0E) [28]. The human and mouse sequences show $92 \%$ sequence identity over 851 amino acids. The models were created using the automatic modelling script with standard parameters of the YASARA \& WHAT IF Twinset $[29,30]$. The resulting model is complete, except for the $\mathrm{N}$-terminal signal peptide.

\section{GANAB constructs}

$G A N A B$ expression clones were generated using the Gateway Cloning system (Thermo Scientific, Carlsbad, CA). Entry clone encoding full-length human $G A N A B$ (2901 bp) was generated from cDNA of liver tissue. Total RNA was isolated from liver tissue using TRIzol Reagent (Thermo Scientific), and cDNA was generated by RT Transcriptor First Strand cDNA synthesis kit (Roche Applied Sciences, Mannheim, Germany) using a $G A N A B$ specific reverse primer. Full-length $G A N A B$ $W T$ fragments were obtained using Platinum ${ }^{\mathrm{TM}} \operatorname{SuperFi}^{\mathrm{TM}}$
DNA Polymerase (Thermo Scientific) using forward and reverse primers including the attB1 and attB2 sequence. The GANAB-WT fragment was then cloned into the Gateway entry vector pDONR201. The entry clones of GANAB including wild type and mutant intron 17 were generated by replacing a BglI/KpnI fragment of pDONR201GANAB-WT by a fragment produced by PCR on patient genomic DNA having a heterozygous intronic mutation using an exon 14 forward and exon 18 reverse primer followed by digestion with BglI and KpnI. All $G A N A B$ entry clones were subsequently cloned into the GW331 N-terminal FLAG expression vector. GANAB constructs harboring exonic variants were generated using the Quick Change-II-XL Site-Directed Mutagenesis Kit (Agilent Technologies, Santa Clara, USA) and the GW331GANAB-WT as a template. Sequences of all constructs were confirmed by Sanger sequencing.

\section{Transfection of cells}

HEK293 and HeLa cells were cultured in DMEM supplemented with $10 \%$ FCS, 1\% NEAA, $50 \mu \mathrm{g} / \mathrm{mL}$ Gentamicin, and $10 \mathrm{mM}$ HEPES (all Thermo Scientific) at $37{ }^{\circ} \mathrm{C}$ and $5 \% \mathrm{CO}_{2}$ in a humidified incubator. For DNA transfections, HEK293 and HeLa cells were seeded in a 6-wells plate $(500,000$ cells per well) for Western blotting and HeLa cells also in a 24-wells plate containing poly-l-lysine coated glasses $(100,000$ cells per well) for immunofluorescent staining. The following day cells were transfected with GW331GANAB constructs using FuGene HD (Promega, Madison, WI). For stable expression of GII $\alpha$, normal medium of cells was replaced by medium containing $500 \mu \mathrm{g} / \mathrm{mL}$ G418 (Thermo Scientific) after 2 days. After 2 weeks resistant clones were transferred to a new well and tested for stable expression of GII $\alpha$ by Western blotting.

\section{Immunofluorescent staining}

Human cholangiocytes or HeLa cells were fixed in $2 \%$ paraformaldehyde and incubated in phosphate buffered saline/1\% Bovine serum albumin/1\% Normal swine serum $/ 0.1 \%$ Cold water fish gelatin containing primary antibodies against PRKCSH (1:100, mouse polyclonal, Santa Cruz Biotechnology, Dallas, TX), GANAB (1:200, rabbit polyclonal, Thermofisher Scientific), FLAG (1:100, rabbit polyclonal, Sigma) or protein disulfide isomerase (PDI, 1:100 mouse monoclonal, Stressgen Biotechnologies, San Diego, CA). Then glasses were incubated with the corresponding secondary fluorescent antibodies Texas Red (1:100, mouse, Jackson Immunoresearch, West Grove, PA), FITC (1:100, rabbit, Jackson Immunoresearch) and DAPI (1:1000, Roche) for nuclear staining. Images were taken using FV1000 Confocal Laser Scanning Microscope (Olympus, Tokio, Japan). 


\section{Western blot analysis}

Cells were lysed using lysis buffer $(50 \mathrm{~mm}$ Tris- $\mathrm{HCl}$ (pH7.5), $150 \mathrm{~mm} \mathrm{NaCl}, 1 \% \mathrm{NP}-40$, protease inhibitor tablet (Roche)) on ice $72 \mathrm{~h}$ after transfection. After adding sample buffer to the lysed cells, samples were heated for $5 \mathrm{~min}$ at $95{ }^{\circ} \mathrm{C}$. Samples were run on a $10 \%$ SDS-PAGE gel and transferred semi dry onto a nitrocellulose membrane. Immunostaining was perfomed with anti-Flag as primary antibody and swine anti-rabbit HRP as a secondary antibody (DAKO, Glostrup, Denmark). Proteins were visualized using Clarity Western ECL Substrate (Biorad) and the Proxima C18 imaging system (Isogen, de Maarn, Netherlands). Western Blots were quantified using Totallab quant (Totallab, Newcastle-Upon-Tyne, United Kingdom).

\section{RNA isolation and RT-qPCR}

Total RNA was isolated using TRIzol according to manufacturer's protocol. We reverse transcribed RNA into complementary DNA (cDNA) using iScript cDNA synthesis kit according to the protocol (Biorad). $1 \mathrm{~mL}$ of resulting cDNA was used for RT-qPCR. Briefly, the RTqPCR was carried out on an thermal cycler (CFX96, Biorad), using the 2_DDCt SYBR green protocol.We amplified GANAB and beta actin (reference) in 40 cycles. RT-qPCR data were analyzed using the CFX-Manager software, which validates primer quality by analyzing melting curves. All the RT-qPCRs were performed with triplicates for each sample.

\section{Supplementary information}

Supplementary information accompanies this paper at https://doi. org/10.1186/s13023-020-01585-4.

Additional file 1. Overview of coverage and Sanger sequencing results of 38 GANAB variants identified by molecular inversion probe analysis

Additional file 2. Protein analysis of wild type and mutant Glla in HeLa cells. Western blot detection using anti-Flag antibody of cell lysate from transiently transfected HeLa cells using N-terminal Flag tagged construct with GANAB Wild Type (WT) and variants C.11_16delTAGCGG (11), c.687delT (687), c.2002+1G>C (INT), c.2509C >T (2509) and c.2656C>T (2656) at the left of the marker (M). The constructs showed similar amounts of expressed Glla with predicted molecular weights

Additional file 3. DNA and protein expression analysis of Glla. DNA expression of Glla Wild Type intron (WT) and mutant intron (MT intron) was analysed using qPCR with B-actin (ACTB) as reference. WT expression was 1.46 times higher than MT intron. On Western Blot expression of WT protein was 4 times higher than MT intron protein. In absolute numbers protein expression of WT intron was 2.67 times higher than MT intron protein

Additional file 4. Localization of wild-type and mutant Glla in HeLa cells. Immunofluorescence staining of cells transfected with GANAB Wild Type (WT) and variants C.11_16delTAGCGG (11), c.687delT (687), c.2002+1G>C (int), c.2509C>T (2509), and c.2626C>T (2656). Glla (green) staining shows normal localization of WT, variant 11, variant 2509, and variant 2656.
Variant 687 shows subcellular localization. Variant int localizes to the nucleus (blue), or localizes subcellular

\section{Abbreviations}

ADPKD: Autosomal dominant polycystic kidney disease; ADPLD: Autosomal dominant polycystic liver disease; ER: Endoplasmic reticulum; Gll: Glucosidase II; Glla: Glucosidase II a-subunit; GIIß: Glucosidase II $\beta$-subunite; MIP: Molecular inversion probe; PC1: Polycystin-1; PKD1: Polycystic kidney disease 1; PKD2: Polycystic kidney disease 2; PLD: Polycystic liver diseasea; PTC: Premature termination codon.

\section{Acknowledgements}

Not applicable.

\section{Authors' contributions}

Study conception was performed by WC, AH, JD and RR. Material preparation, data collection and analysis were performed by $L L, R M, A H, H V, H R, W C$ and JB. The first draft of the manuscript was written by $L L$ and all authors commented on previous versions of the manuscript. All authors read and approved the final manuscript.

\section{Funding}

This research was funded by a grant of the Radboud Institute for Molecular Life Sciences which had no role in the design of the study and collection, analysis, and interpretation of data and in writing the manuscript.

\section{Availability of data and material}

All new variations found in this study were submitted to the ClinVar database with numbers RCV000758153 to RCV000758162.

\section{Ethics approval and consent to participate}

This study was performed in line with the principles of the Declaration of Helsinki. Approval was granted by the Ethics Committee of the Radboud University Medical Center (CMO regio Arnhem-Nijmegen; 2001-218). All participants included in the study gave informed consent.

\section{Consent for publication}

Not applicable.

\section{Competing interests}

The authors declare that they have no competing interests.

\section{Author details}

${ }^{1}$ Department of Gastroenterology and Hepatology, Institute for Molecular Life Sciences, Radboud University Medical Center, P.O. Box 9101, 6500 HB Nijmegen, The Netherlands. ${ }^{2}$ Department of Human Genetics, Institute for Molecular Life Sciences, Radboud University Medical Center, Nijmegen, The Netherlands. ${ }^{3}$ Centre for Molecular and Biomolecular Informatics, Institute for Molecular Life Sciences, Radboud University Medical Center, Nijmegen, The Netherlands. ${ }^{4}$ Department of Liver and Gastrointestinal Diseases, Biodonostia Research Institute - Donostia University Hospital, University of the Basque Country (UPV/EHU), San Sebastián, Spain. ${ }^{5}$ IKERBASQUE, San Sebastián, Spain. ${ }^{6}$ CIBERehd, San Sebastián, Spain.

Received: 10 May 2020 Accepted: 13 October 2020

Published online: 23 October 2020

\section{References}

1. Hoevenaren IA, Wester R, Schrier RW, McFann K, Doctor RB, Drenth JP, et al. Polycystic liver: clinical characteristics of patients with isolated polycystic liver disease compared with patients with polycystic liver and autosomal dominant polycystic kidney disease. Liver Int. 2008;28(2):264-70.

2. Cnossen WR, Drenth JP. Polycystic liver disease: an overview of pathogenesis, clinical manifestations and management. Orphanet J Rare Dis. 2014;9:69. 
3. Mikolajczyk AE, Te HS, Chapman AB. Gastrointestinal manifestations of autosomal-dominant polycystic kidney disease. Clin Gastroenterol Hepatol. 2017;15(1):17-24

4. Perugorria MJ, MasyukTV, Marin JJ, Marzioni M, Bujanda L, LaRusso NF, et al. Polycystic liver diseases: advanced insights into the molecular mechanisms. Nat Rev Gastroenterol Hepatol. 2014;11(12):750-61.

5. Van Keimpema L, De Koning DB, Van Hoek B, Van Den Berg AP, Van Oijen MG, De Man RA, et al. Patients with isolated polycystic liver disease referred to liver centres: clinical characterization of 137 cases. Liver Int. 2011;31(1):92-8

6. Fedeles SV, Tian X, Gallagher AR, Mitobe M, Nishio S, Lee SH, et al. A genetic interaction network of five genes for human polycystic kidney and liver diseases defines polycystin-1 as the central determinant of cyst formation. Nat Genet. 2011:43(7):639-47.

7. Besse W, Dong K, Choi J, Punia S, Fedeles SV, Choi M, et al. Isolated polycystic liver disease genes define effectors of polycystin-1 function. J Clin Invest. 2017;127:1772-85.

8. Waanders E, te Morsche RH, de Man RA, Jansen JB, Drenth JP. Extensive mutational analysis of PRKCSH and SEC63 broadens the spectrum of polycystic liver disease. Hum Mutat. 2006:27(8):830

9. Cnossen WR, te Morsche RH, Hoischen A, Gilissen C, Chrispijn M, Venselaar $\mathrm{H}$, et al. Whole-exome sequencing reveals LRP5 mutations and canonical Wnt signaling associated with hepatic cystogenesis. Proc Nat Acad Sci USA. 2014;111(14):5343-8.

10. Wilson EM, Choi J, Torres VE, Somlo S, Besse W. Large Deletions in GANAB and SEC63 explain 2 cases of polycystic kidney and liver disease. Kidney Int Rep. 2020:5(5):727-31.

11. Treml K, Meimaroglou D, Hentges A, Bause E. The a- and $\beta$-subunits are required for expression of catalytic activity in the hetero-dimeric glucosidase II complex from human liver. Glycobiology. 2000a;10(5):493-502.

12. Satoh T, Toshimori T, Yan G, Yamaguchi T, Kato K. Structural basis for two-step glucose trimming by glucosidase II involved in ER glycoprotein quality control. Sci Rep. 2016;6:20575.

13. Drenth JP, Martina JA, van de Kerkhof R, Bonifacino JS, Jansen JB. Polycystic liver disease is a disorder of cotranslational protein processing. Trends Mol Med. 2005;11(1):37-42.

14. Dell'Angelica EC. The building BLOC $(\mathrm{k})$ s of lysosomes and related organelles. Curr Opin Cell Biol. 2004;16(4):458-64.

15. Porath B, Gainullin VG, Cornec-Le Gall E, Dillinger EK, Heyer CM, Hopp $K$, et al. Mutations in GANAB, encoding the glucosidase iialpha subunit, cause autosomal-dominant polycystic kidney and liver disease. Am J Hum Genet. 2016:98(6):1193-207.

16. Peces R, Drenth JPH, Te Morsche RHM, González P, Peces C. Autosomal dominant polycystic liver disease in a family without polycystic kidney disease associated with a novel missense protein kinase $C$ substrate 80K-H mutation. World J Gastroenterol. 2005;11(48):7690-3.
17. Satoh T, Toshimori T, Noda M, Uchiyama S, Kato K. Interaction mode between catalytic and regulatory subunits in glucosidase II involved in ER glycoprotein quality control. Protein Sci. 2016;25(11):2095-101.

18. Carrim Zl, Murchison JT. The prevalence of simple renal and hepatic cysts detected by spiral computed tomography. Clin Radiol. 2003;58(8):626-9.

19. Pelletier MF, Marcil A, Sevigny G, Jakob CA, Tessier DC, Chevet E, et al. The heterodimeric structure of glucosidase II is required for its activity, solubility, and localization in vivo. Glycobiology. 2000;10(8):815-27.

20. Treml K, Meimaroglou D, Hentges A, Bause E. The alpha- and betasubunits are required for expression of catalytic activity in the heterodimeric glucosidase II complex from human liver. Glycobiology. 2000b;10(5):493-502

21. Roth J, Zuber C. Quality control of glycoprotein folding and ERAD: the role of N-glycan handling, EDEM1 and OS-9. Histochem Cell Biol. 2017;147(2):269-84.

22. Fedeles SV, Gallagher AR, Somlo S. Polycystin-1: a master regulator of intersecting cystic pathways. Trends Mol Med. 2014;20(5):251-60.

23. Hopp K, Ward CJ, Hommerding CJ, Nasr SH, Tuan HF, Gainullin VG, et al. Functional polycystin-1 dosage governs autosomal dominant polycystic kidney disease severity. J Clin Invest. 2012;122(11):4257-73.

24. Gainullin VG, Hopp K, Ward CJ, Hommerding CJ, Harris PC. Polycystin-1 maturation requires polycystin-2 in a dose-dependent manner. J Clin Invest. 2015;125(2):607-20.

25. Hofherr A, Wagner C, Fedeles S, Somlo S, Kottgen M. N-glycosylation determines the abundance of the transient receptor potential channel TRPP2. J Biol Chem. 2014;289(21):14854-67.

26. D'Agnolo HM, Kievit W, Andrade RJ, Karlsen TH, Wedemeyer H, Drenth JP. Creating an effective clinical registry for rare diseases. United Eur Gastroenterol J. 2016:4(3):333-8.

27. Khandelwal KD, Ishorst N, Zhou H, Ludwig KU, Venselaar H, Gilissen C, et al. Novel IRF6 mutations detected in orofacial cleft patients by targeted massively parallel sequencing. J Dent Res. 2016;96(2):179-85.

28. Caputo AT, Alonzi DS, Marti L, Reca I-B, Kiappes JL, Struwe WB, et al. Structures of mammalian ER a-glucosidase II capture the binding modes of broad-spectrum iminosugar antivirals. Proc Natl Acad Sci USA. 2016:113(32):E4630-8.

29. Krieger E, Koraimann G, Vriend G. Increasing the precision of comparative models with YASARA NOVA — a self-parameterizing force field. Proteins. 2002;47(3):393-402.

30. Vriend G. WHAT IF: a molecular modeling and drug design program. J Mol Graph. 1990;8(1):52-6.

\section{Publisher's Note}

Springer Nature remains neutral with regard to jurisdictional claims in published maps and institutional affiliations.
Ready to submit your research? Choose BMC and benefit from:

- fast, convenient online submission

- thorough peer review by experienced researchers in your field

- rapid publication on acceptance

- support for research data, including large and complex data types

- gold Open Access which fosters wider collaboration and increased citations

- maximum visibility for your research: over 100M website views per year

At BMC, research is always in progress.

Learn more biomedcentral.com/submissions 